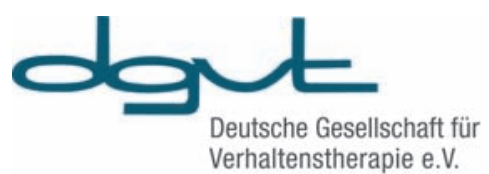

\section{Deutsche Gesellschaft für Verhaltenstherapie (DGVT) e.V.}

\author{
Bundesgeschäftsstelle \\ Corrensstraße 44-46 \\ 72076 Tübingen, Deutschland \\ Tel. +49 7071 9434-0, Fax -35 \\ dgvt@dgvt.de,www.dgvt.de
}

\section{Expertinnen-Interview mit Dr. Silja Samerski, Universität Bremen: Nutzen und Risiken von psy- chotherapeutischen E-Health-Angeboten}

Anbei in Auszügen ein Interview mit Dr. Silja Samerski, Wissenschaftliche Mitarbeiterin am Institut für Ethnologie und Kulturwissenschaft an der Universität Bremen (internationales Forschungsprojekt zu Gesundheit, Wohlfahrt und Superdiversity). Das schriftliche Interview für den DGVT-Berufsverband führte Dr. Pia Beiderwellen, Psychologische Psychotherapeutin aus Dortmund.

12. Februar 2018

1. Die zunehmende Digitalisierung des Gesundheitswesens ist nicht nur in technischer Hinsicht ein komplexes Geschehen, sondern erfordert auch eine Auseinandersetzung mit den damit verbundenen rechtlichen Aspekten. Könnten Sie uns über die aktuelle Debatte einen kurzen Überblick geben?

Im Zentrum der aktuellen rechtlichen Debatte steht der Schutz der Persönlichkeit bzw. der Datenschutz. Das deutsche Datenschutzrecht erlaubt das zweckfreie Sammeln von Daten eigentlich nicht - und steht damit im Widerspruch zu den heutigen Praktiken des Datensammelns. Vor fast 35 Jahren hat das Bundesverfassungsgericht (BVerfG) das Recht auf informationelle Selbstbestimmung formuliert, also das Recht, selbst über die Preisgabe von persönlichen Daten bestimmen zu können. Die Formulierungen sind heute aktueller denn je: Das BVerfG stellte fest, dass «mit dem Recht auf informationelle Selbstbestimmung (...) eine Gesellschaftsordnung nicht vereinbar (wäre), in der Bürger nicht mehr wissen können, wer was wann und bei welcher Gelegenheit über sie weiß.» Sich frei entfalten und frei mitwirken im demokratischen Gemeinwesen können nur diejenigen, die nicht davon ausgehen müssen, dass ihr Tun überwacht und gespeichert wird, so das BVerfG. Heute passiert jedoch genau das, immer mehr Lebensäußerungen werden registriert und gespeichert. Die größten Datensammler sind jedoch in Deutschland nicht staatliche Institutionen, sondern die großen Konzerne. Verfechter von Big Data versuchen nun, das Recht auf informationelle Selbstbestimmung «weiterzuentwickeln», wie der Deutsche Ethikrat es formuliert - «aufzuweichen» wäre wohl treffender. Meist wird leider die informationelle Selbstbestimmung durchlöchert, um Big Data und Digitalisierung nicht zu behindern - und nicht umgekehrt. Die Europäische Datenschutz-Grundverordnung, die im Mai 2018 in Kraft tritt, versucht ja, die informationelle Selbstbestimmung gegenüber den allgegenwärtigen Datenkraken zu verteidigen. Soweit ich gehört habe, wird das Gesetz bereits jetzt schon ausgehöhlt. Daten sind das Öl des 21. Jahrhunderts, das muss man sich klarmachen.

2. In Deutschland ist die Online-Psychotherapie unter anderem wegen der rechtlichen Bedenken (vgl. unter anderem die Berufsordnungen der Psychotherapeutenkammern) noch nicht Bestandteil der Regelversorgung, sondern wird derzeit lediglich im Rahmen von Forschungsprojekten angeboten. Gibt es Erfahrungswerte aus der Regelversorgung in anderen Ländern, die auf unsere Versorgungsstruktur übertragbar sind?

Ich weiß, dass in vielen anderen Ländern, z.B. in den USA oder in Großbritannien, Online-Psychotherapien bereits im Einsatz sind. Die Gesundheitssysteme sind dort aber völlig anders; Großbritannien hat eine zentrale staatliche Gesundheitsversorgung, den NHS, und «Psychotherapeut» ist z.B. keine geschützte Bezeichnung. Online-Psychotherapien werden sowohl in Großbritannien als auch in den USA offenbar in wachsendem Maße nachgefragt. Ein Grund dafür sind auch dort die langen Wartezeiten für eine herkömmliche Psychotherapie.

Was aus den dortigen Berichten deutlich wird ist erstens, dass Therapeuten für textbasierte Online-Therapien unbedingt geschult werden müssen. Das Schreiben erfordert andere Umgangsweisen und Sensibilitäten und führt schnell zu Missverständnissen. Daher gibt es in Großbritannien schon das Zertifikat zum «Certified Cyber Therapist». Zweitens wird immer wieder betont, dass reine OnlineTherapien eigentlich nicht erstrebenswert sind. Es ist wichtig, dass ein Arzt oder Therapeut den Patienten auch zu Gesicht bekommt. In einem Artikel merkt ein Patient - eigentlich ein Technik-Fan - 
an, dass ihm erst die Online-Sitzung deutlich gemacht habe, wie wichtig die zwischenmenschliche Begegnung ist.

3. Wenn die Datensicherheit der patientenbezogenen Informationen auch in einer digitalen Gesundheitsbranche gewährleistet wäre, welche weiterführenden ethischen und sozialen Belange sind bei der Digitalisierung zu benennen und zu berücksichtigen? Unterscheidet sich das psychotherapeutische Handlungsfeld hierbei von anderen heilkundlichen Bereichen?

Ich will vor allem einen Aspekt nennen, der in der herrschenden Diskussion oft untergeht: Die Frage, wie sich unser Gesundheitswesen und letztlich unser soziales Miteinander durch die Digitalisierung ändert. Die Psychotherapie ist ja weitgehend eine sprechende Profession; der Patient spricht von sich, und die Psychotherapeutin hört zu. Die beiden gehen eine Beziehung miteinander ein, in der es um viel mehr geht als um die Anwendung von Techniken. Der Sinn dafür, dass ein solches Gespräch, in dem zwei Menschen leiblich gegenwärtig sind, etwas völlig anderes ist als die Kommunikation von Information - Information austauschen kann auch ein Computer - geht in unserer Gesellschaft zunehmend verloren. Bis zum Anfang des 18. Jahrhunderts war jede Begegnung zwischen Arzt und Patient eine Konversation. Patienten erzählten von ihren Leiden und vertrauten darauf, dass ihre Erzählungen im Arzt einen Widerhall fanden. Die medizinische Kunst bestand vornehmlich im Zuhören. Die Verwissenschaftlichung der Medizin und Objektivierung des menschlichen Körpers haben den Patienten in der Medizin jedoch zum Verstummen gebracht. Das Anhören wurde sozusagen durch das Abhorchen abgelöst. In einer datengetriebenen Medizin verschwindet nun auch der individuelle Körper und wird durch ein Datenprofil ersetzt. Was als normal und was als auffällig bzw. behandlungsbedürftig gilt, bestimmen Daten, Normwerte und statistische Korrelationen - ganz gleich, wie sich der leibhaftige Mensch fühlt und woran er leidet. Vor ein paar Jahren beklagte ein Teilnehmer auf einem Ärztekongress, dass die Medizin anfange, nicht mehr Menschen zu behandeln, sondern die «Grafiken».

Für mich ist die zentrale Frage angesichts der Digitalisierung, ob es gelingen kann, das Bewusstsein für die Kluft zwischen virtueller und realer Welt zu kultivieren: die Kluft zwischen gesichtslosem Datenprofil und leibhaftiger Person, zwischen Risikokalkulation und unvorhersehbarer Zukunft, zwischen Statistik und unberechenbarem Individuum, zwischen technisch vermittelter Kommunikation und zugewandtem Gespräch. Vielleicht können Psychotherapeuten hier Vorreiter sein, weil sie noch wissen, wie wichtig Gegenwart, Zuhören und Verstehen für eine menschliche Begegnung und Heilkunst sind.

\section{$(\ldots)$}

6. Ein weiteres ethisches Prinzip, welches sich in verschiedenen ethischen Richtlinien findet, ist das Prinzip der Verantwortung. Der Verbraucherzentrale Bundesverband e.V. appelliert in Bezug auf die Einrichtung einer Online-Sprechstunde an die Patientinnen und Patienten, bei ihrem Arzt Druck zu machen. Wer trägt
Ihrer Meinung nach die Verantwortung für die Etablierung von Online-Angeboten?

Verantwortung ist heute ein großes und zugleich leeres Wort; es ist höchst aufgeladen, ohne etwas Konkretes zu bedeuten. Meist wird es appellativ gebraucht, es hat vor allem eine soziale Funktion. Ich erinnere mich beispielsweise noch, dass mich Genetiker für den Tod von Kranken verantwortlich machen wollten, weil ich die Gentechnik kritisierte. Letztlich kann ich ja nur für das Verantwortung übernehmen, was in meinem Einflussbereich liegt. Wie gesagt, Online-Angebote müssen geprüft und zertifiziert werden, und hierfür sind dann die entsprechenden deutschen Behörden verantwortlich, z.B. das Bundesinstitut für Arzneimittel und Medizinprodukte sowie die Datenschutzbeauftragten. Ansonsten würde ich nicht von Verantwortung sprechen, sondern die Etablierung von Online-Angeboten - idealerweise - als gesellschaftlichen Aushandlungsprozess verstehen, bei dem verschiedene Gruppen, Interessen, Vorstellungen und Perspektiven sich miteinander sowie mit den technologischen Entwicklungen und ihren Folgen auseinandersetzen. Das braucht seine Zeit, aber so ist das eben mit demokratischen Vorgängen.

Das vollständige Interview finden Sie unter folgendem Link: www.dgvt.de/fileadmin/user_upload/DGVT-BV/Dokumente/ Experteninterview_mit_Frau_Samerski_Antworten_final.pdf.

\section{Fort- und Weiterbildung}

Die 17. Praxistage der Kinder- und Jugendlichenpsychotherapie werden vom 3.-4. November 2018 in Münster stattfinden. Zahlreiche Workshops werden sich dort mit dem Thema «Am Limit Therapeutische Grenzsituationen» auseinandersetzen. Dr. Wilhelm Rotthaus gibt in seinem Eröffnungsvortrag einen Überblick zum Thema «Jugendliche und TherapeutInnen am Limit».

Vom 15.-16. September 2018 findet die 6. Tagung Psychotherapie-State-of-the-Art in Potsdam am Neuen Palais statt. Das Thema der Tagung lautet in diesem Jahr «Aktuelle Ansätze der Traumatherapie». Prof. Dr. med. Ingo Schäfer wird in seinem Eröffnungsvortrag einen aktuellen Stand zur evidenzbasierten Behandlung von Traumastörungen geben. Die 2-tägigen Workshops befassen sich mit den Themen «STAIR/NT - ein Therapieprogramm zur Behandlung der Folgen von sexueller und körperlicher Gewalt in der Kindheit», "Dialektisch Behaviorale Therapie der Posttraumatischen Belastungsstörungen nach interpersoneller Gewalt in der Kindheit (DBT-PTSD)», «Cognitive Processing Therapy $(\mathrm{CPT})$ » und «Einführung in die Narrative Expositionstherapie $(\mathrm{NET}) »$.

Ausführliche Informationen zu diesen und weiteren Angeboten erhalten Sie unter www.dgvt-fortbildung.de oder über die DGVTBundesgeschäftsstelle in Tübingen, Tel.: 070719434 34, Fax: 07071 9434 35, E-Mail: fortbildung@dgvt.de. Sprechzeiten: Di und Do 9:00-12:00 Uhr; Di und Mi 14:00-15:30 Uhr. 


\section{Deutsche Gesellschaft für Verhaltensmedizin und Verhaltensmodifikation (DGVM)}

Prof. Dr. Claus Vögele

Institute for Health and Behaviour

Research Unit INSIDE

University of Luxembourg - Campus Belval

Maison des Sciences Humaines

11, Porte des Sciences

4366 Esch-sur-Alzette, Luxemburg

- Erstmals wurden die Förderpreise im Rahmen des neu initiierten Peer-Mentoring-Programms der DGVM vergeben. Aus den eingegangenen Anträgen wurden 3 Bewerbungen zur Förderung ausgewählt: Hannes Noack (Universität Tübingen) und Nadine Skoluda (Universität Marburg); Marcel N. Jarczok (Universitätsklinikum Ulm) und Katja Schmalenberger (Universität Heidelberg); Sarah Schumacher (FU Berlin) und Susanne Fischer (Universität Zürich).

- Studie zu Frauengesundheit 40+ (Serena Fiacco, Laura Mernone und Ulrike Ehlert; Universität Zürich):

Insgesamt 245 gesunde Frauen zwischen 40 und 73 Jahren haben an der Studie "Frauengesundheit 40+» teilgenommen. Der Fokus der Studie ist eine biopsychosoziale Untersuchung von Gesundheit und Faktoren gesunden Alterns. Die Studie ist ein Teilprojekt des Forschungsschwerpunkts «Dynamik Gesunden Alterns». Weitere Informationen finden sich unter $w w w$. frauengesundheit40plus.ch.

- Männerstudie 40+ (Andreas Walther, Tim Lacker und Ulrike Ehlert, Universität Zürich):

Die Ersterhebung der Studie «Männergesundheit 40+ - Biopsychosoziale Faktoren gesunden Alterns», eines Teilprojekts des universitären Forschungsschwerpunkts «Dynamik Gesunden Alterns» wurde mit 271 Männern erfolgreich abgeschlossen. Weitere Informationen auf: www.männer40plus.ch.

- Ulrike Ehlert (Universität Zürich) ist Mitglied der AG Pathophysiologie und Genetik der S3-Leitlinienkommission Reizdarmsyndrom.

- Christina Hunger-Schoppe (Heidelberg) vertritt im Sommersemester 2018 die Professur für Gesundheitspsychologie und Angewandte Diagnostik an der Bergischen Universität Wuppertal.

\section{$M$}

\author{
AVM Deutschland \\ Arbeitsgemeinschaft für Verhaltensmodifikation e.V. (AVM-D) \\ Bundesgeschäftsstelle \\ Promenadestraße 8, 96047 Bamberg, Deutschland \\ Tel. +499512085211 \\ info@avm-d.de, \\ info@avm-d.de,www.avm-d.de,www.avm-institute.de
}

\section{Ab Herbst 2018 \\ Start der Ausbildungen zu Psychologischen PsychotherapeutInnen und Kinder- und JugendlichenpsychotherapeutInnen}

Im Oktober 2018 starten unsere neuen Ausbildungsgänge für Psychologische PsychotherapeutInnen (PP) und Kinderund JugendlichenpsychotherapeutInnen (KJP) an diesen AVMAusbildungsstandorten:

- Bamberg

- München

- Nürnberg

- Neu-Ulm

- Regensburg

- Stuttgart

- Würzburg

Bewerbungen sind ab sofort möglich. Weitere Informationen sowie die Termine für unsere Infoveranstaltungen finden Sie auf unserer Homepage: www.avm-institute.de oder auf Facebook: www.facebook.com/avm.deutschland.

\section{Fort- und Weiterbildung:}

\section{Training Sozialer Kompetenzen (TSK)}

\section{Mehr Selbstsicherheit und soziale Kompetenz}

\section{Workshop-Reihe mit DP Erika Güroff}

Schwierigkeiten im Umgang mit anderen Menschen sind weit verbreitet und breit gefächert. Viele Ängste kennen wir alle. Wenn Ängste aber das Erleben dominieren und das Lebensgefühl verdüstern, sind sie immer gekoppelt mit sehr typischen Verhaltensunsicherheiten, die wir Störungen der sozialen Kompetenz nennen. In der psychotherapeutischen Praxis ist die Diagnose bei solchen Ängsten und Unsicherheiten häufig eine soziale Phobie oder in schweren 
Fällen die ängstlich vermeidende Persönlichkeitsstörung. Aber auch bei vielen anderen psychischen Störungen sind soziale Ängste und Verhaltensunsicherheiten ein wesentliches, wenn auch oft verdecktes Element, z.B. bei Depression, Burnout oder Panikstörung.

Die Aufgabe der PsychotherapeutInnen ist, Vermeidungs- und Ausweichstrategien herauszuarbeiten und in das Behandlungssetting aufzunehmen. Das Ziel des TSK ist es, den PatientInnen mit klassischen VT-Methoden, wie z.B. hierarchisch aufgebauten Rollenspielen, Modelllernen, Videotraining, Feedback oder Hausaufgaben, Selbstsicherheit und soziale Kompetenz zu vermitteln.

Als Workshop-Material erhalten Sie das Trainingsprogramm «Selbstsicherheit und soziale Kompetenz» mit beiliegender DVD von Frau Güroff.

Termine und Orte:

Regensburg: 27./28. Oktober, 17./18. November und

8./9. Dezember 2018

München: 10./11. November, 15./16. Dezember 2018 und

19./20. Januar 2019

\section{Psychoonkologie}

\section{Menschen mit Krebs begleiten}

\section{Workshop mit DP Claudia Erzberger}

Die Psychotherapie bei Menschen mit Krebserkrankung bringt vielseitige Fragen und Herausforderungen mit sich. Betroffene und Angehörige suchen nach einem erträglichen Umgang mit Schmerz, Abschiednehmen, Sterben und Tod. Langanhaltende Ängste bedürfen häufig der therapeutischen Aufarbeitung in dieser besonderen Belastungssituation. Dipl.-Psych. Claudia Erzberger, die den Psychoonkologischen Konsiliardienst am Klinikum Bogenhausen (StKM) leitet, geht in diesem Workshop intensiv auf Besonderheiten in der Arbeit mit krebskranken Menschen ein und zeigt konkrete Herangehensweisen für die therapeutische Begleitung. Fallbeispiele und konkrete Anregungen für die eigene Arbeit sollen die TeilnehmerInnen dabei unterstützen, sich für die Behandlung krebskranker Menschen gut gerüstet zu fühlen.

Termin und Ort:

München: 14. Juli 2018

\section{"Ich packe meinen (Methoden-)Koffer...":}

\section{Systemische Methoden erleben und erleben lassen}

Workshop-Reihe mit Katja Zenz, Pädagogion M.A.

Die Systemische Therapie hat viele Wurzeln und präsentiert sich zwischenzeitlich mit verschiedensten Ansätzen, wie z.B. dem strukturellen, narrativen, lösungsorientierten oder generationalen
Ansatz und vielen mehr. Einheitlichkeit zeigt sich meist dort, wo es um das Verständnis von Krankheit und Methodenvielfalt geht: Die Symptomatik wird als Lösungsansatz verstanden, wenn auch als langfristig dysfunktionaler. Es geht nicht um eine Diagnose, sondern um das Verständnis des Symptoms in dem jeweiligen Kontext. Dabei wird als Methode eingesetzt, was hilft.

An den beiden, voneinander unabhängigen Wochenenden bietet Ihnen Katja Zenz an, ganz praxisnah und mit einem gewissen $\mathrm{Maß}$ an Selbsterfahrung verschiedene systemische Methoden wie z.B. zirkuläres Fragen, Wunderfrage, Aufstellungsarbeit, Reframing, paradoxe Intervention und vieles mehr kennenzulernen. Dabei wird Ihnen neben der technischen Anwendung vor allem die Möglichkeit gegeben, sich mithilfe dieser Methoden eigenen Fragestellungen anzunähern.

Termine und Orte:

München: 22./23. September 2018

Würzburg: 20./21. Oktober 2018

\section{Bewusstheit, Mut und therapeutische Liebe}

\section{Die Funktional Analytische Psychotherapie (FAP)}

\section{Workshop-Wochenende mit DP Norbert Schneider}

Die Funktional-Analytische Psychotherapie (FAP) ist eine intime, emotional intensive kontextuelle Verhaltenstherapie, in der die therapeutische Beziehung als zentrales Vehikel für Veränderung dient. FAP-Therapeuten achten darauf, wie die sozialen und emotionalen Probleme und Fortschritte ihrer Klienten im Hier und Jetzt der therapeutischen Begegnung in Erscheinung treten (Bewusstheit).

Sie versuchen, sozial und emotional herausfordernde Situationen in der Therapiesitzung zu aktualisieren, an denen ihre Klienten (und auch sie selbst) wachsen können (Mut). Sie bemühen sich, authentische und intensive therapeutische Beziehungen $\mathrm{zu}$ entwickeln (therapeutische Liebe), um ihre Klienten zu befähigen, auch jenseits des Therapieraums erfüllende und intime Beziehungen zu entwickeln.

In praxisnahen Reflexionen sowie über das eigene Erleben werden Antworten gesucht auf Fragen wie: Wie gehen wir damit um, wenn sich das «Problemverhalten» der Klienten auch in der Therapiesitzung zeigt? Wie können wir herausfordernde Situationen in der Therapie herstellen, an denen unsere Klienten (und auch wir selbst) wachsen? Wie können wir offene, authentische und «reale» Beziehungen mit unseren Klienten entwickeln? Wie viel dürfen wir von uns selbst in der Therapiebeziehung zeigen? Wie gehen wir als Therapeuten mit unserer eigenen Verletzlichkeit um?

Termin und Ort:

München: 1./2. Dezember 2018 


\section{ACT - Acceptance and Commitment Therapy}

Workshop-Reihe mit Dr. Nathali Klingen

ACT - eine Therapieform, die ganz neue Wege einschlägt. Das Ziel: Ein zufriedenes und erfülltes Leben, trotz und mit bestehenden psychischen (und anderen) Problemen. Nicht die Überwindung der Symptome steht im Vordergrund, sondern die persönlichen Werte und Ziele des Patienten.

Mit verschiedenen erlebnisorientierten Techniken, Metaphern und einer intensiven therapeutischen Beziehung wird der Patient befähigt, psychisch flexibler zu werden und ein werteorientiertes Leben zu führen - unabhängig von seiner derzeitigen Symptomatik. Die ACT gehört zu den führenden Therapieansätzen aus der «dritten Welle» der Verhaltenstherapie, sie ist bei unterschiedlichsten Krankheiten einsetzbar und hat sich - mittlerweile in zahlreichen Studien überprüft - als äußerst wirksam erwiesen.

An den 3 Workshop-Tagen stellt Dr. Nathali Klingen Grundlagen und Anwendungen der ACT dar. Sie erläutert zuerst das Modell der 6 Kernprozesse (Hexaflex) und vermittelt zentrale ACTStrategien und Metaphern. In praktischen Übungen wird die Wirkung dieser Achtsamkeits- und Akzeptanzfertigkeiten selbst erlebt.

Termine und Ort:

München: 12. Oktober, 9.November und 7. Dezember 2018

\section{Weitere Informationen}

Für unsere Fort- und Weiterbildungen werden PTK-Punkte beantragt, Sie erhalten außerdem ein AVM-Zertifikat. Für AVMMitglieder gelten ermäßigte Workshop-Preise.

Mehr zu unserem Fortbildungsprogramm und den Inhalten finden Sie auf unserer Homepage: www.avm-institute.de/fortbildung.

Sie haben weitere Fragen zu unseren Fort- und Weiterbildungen oder Interesse an einem speziell auf Sie zugeschnittenen Inhouse-Seminar in Ihrer Einrichtung? Rufen Sie uns an - Telefon: 0941 5993599-20 - oder schreiben Sie uns an fortbildung@avm-d.de.

\section{AK-LT-Konferenz und Mitgliederversammlung 2018}

Die nächste AK-LT-Konferenz startet am 23. November und findet in Würzburg statt. Allen AK-Leitern und Supervisoren geht hierzu eine gesonderte Einladung zu.

Am selben Wochenende, und zwar am Samstag, den 24. November 2018, findet auch die nächste Mitgliederversammlung des AVM e.V. in Würzburg statt. Eine Einladung und weitere Informationen gehen allen Mitgliedern noch gesondert zu. Bitte stellen Sie Anträge schriftlich bis 6 Wochen vor der Mitgliederversammlung an den Vorstand.

\section{$M$}

\section{AVM Österreich}

Arbeitsgemeinschaft für Verhaltensmodifikation (AVM)

Paris-Lodron-Straße 32, 5020 Salzburg, Österreich

Tel. +43662884166

Neu: Büro in Wien

Leidesdorfgasse 11-13/Stiege 1/Top 8, 1190 Wien, Österreich

Tel. +4313204132

office@verhaltenstherapie-avm.at,

www.verhaltenstherapie-avm.at

www.tagung-avm.at

\section{0. wissenschaftliche Tagung der AVM: "Verhaltenstherapie: Wir behandeln Menschen, nicht die Diagnose»}

Springer-Schlössl, Wien, 18.-21. Oktober 2018

Wenn Menschen ein Problem haben, versuchen sie entweder für dieses selbst eine Lösung zu finden, oder sie begeben sich in die Hände von Fachleuten. Dadurch ändern sich jedoch oft die Sichtweisen auf ihre Probleme und die eigene Rolle. Besteht dieses Problem z.B. im Kauf eines Computers, so wird der Mensch als «Kundin/Kunde» zum/zur Fachmann/Fachfrau im Elektronikmarkt gehen. Dort erhält er fachgerechte Beratung und Hilfe für die Lösung seines Problems. Geht er ins «Gesundheitssystem», wird er zur/zum Patientin/Patienten, und die/der Gesundheitsexpertin/ -experte (Arzt, Psychologe, Psychotherapeut) macht ihn zur/zum Patienten/Patientin, der/die eine Behandlung erfährt und sich dieser «unterwerfen» soll.

Immer wieder ergeben sich dabei Diskussionen hinsichtlich der Diagnostik und Behandlung psychischer Störungen zwischen den Behandelnden und den Behandelten. Oft wird dabei der dahinterstehende Mensch mit seinen Bedürfnissen, Wünschen, Rollen, seiner Persönlichkeit, aber auch seinen Problemen übersehen. Er wird mit einer Diagnose in die Rolle «Patient/Patientin» gepresst und muss sich Behandlungsstrategien unterwerfen, die sich primär an evaluierten Behandlungskonzepten orientieren.

Das ist zwar «State of the Art», jedoch ist der Mensch mehr als nur sein Problem, und er hat mehrere Rollen und Bedürfnisse als nur «brave/r, kooperative/r und motivierte/r» Patient/Patientin zu sein und «gesund» zu werden.

Die diesjährige Tagung der AVM in Wien rückt diese Problematik in den Mittelpunkt. Das Tagungsthema «Wir behandeln Menschen, nicht die Diagnose» soll das charakterisieren.

Im Mittelpunkt steht entsprechend der Philosophie der AVM der Mensch. 
Das Verhalten und Erleben dieses Menschen wird dabei als Zusammenspiel und Resultat von biologischen, psychologischen, kognitiven, emotionalen, sozialen und kontextuellen Faktoren gesehen. Die Grundlage hierfür sind Lernprozesse auf allen Ebenen seit der Kindheit oder möglicherweise auch schon davor. Diese beeinflussen die Entwicklung eines Menschen bereits bei seiner Geburt, führen weiter zur Ausformung seiner Grundpersönlichkeit, aber auch zu weiteren Entwicklungs-, Anpassungs- und Adaptationsprozessen bzw. der Auseinandersetzung mit und der Verarbeitung von Lebenskrisen und Veränderungen. Normales Verhalten bzw. psychische Gesundheit besteht, wenn dadurch ein ausgewogenes Ausmaß an Wohlbefinden und Zufriedenheit erreicht wird bzw. die vorhandenen Verhaltensressourcen ausreichen, um sich an neue Bedingungen durch multifaktorielle Lernprozesse (physiologisch, kognitiv, emotional, sozial, motorisch) erfolgreich anzupassen. Das beinhaltet ein Menschenbild, nach dem das Individuum in der Lage ist, neue Erfahrungen zu erwerben, in neuen Umwelten sicher zu handeln, neue Strategien zu erwerben und biografische Erlebnisse und Erfahrungen kognitiv und emotional neu zu bewerten, zu verarbeiten, (neuro-)biologisch zu speichern und in neuen Situationen anzuwenden [Gatterer 2018, in press].

An diesem Menschenbild orientieren sich auch die Vorträge der Tagung zu den Themen:

- Einblicke in die Schematherapie mit Kindern;

- Psychotherapie mit Menschen mit leichter Alzheimer-

Demenz und deren Angehörigen;

- DBT-Skills-Training;

- Psychoonkologie - Bedürfnisorientierte Bewältigungsstrategien;

- (Un-)gewöhnliche sexuelle Vorliebe oder gefährliche Präferenzstörung? - Sexualität im Kontext paraphiler Phantasien und Verhaltensweisen;

- Innere-Kind-Arbeit - Was bietet die IRRT?;

- Einführung in das KOMKOM-Kommunikationstraining für Paare.

Die Tagung versucht, eine neue Sichtweise auf Menschen mit psychischen Problemen und Erkrankungen zu entwickeln und daraus individuelle, bedürfnisorientierte Behandlungsmodelle abzuleiten. Dadurch soll auch eine bessere Adhärenz bei der Durchführung von Behandlungsstrategien erreicht werden. Die Kooperation und Kommunikation zwischen Behandler und Behandeltem hinsichtlich der Problemdefinition, Diagnosefindung, Motivation zur Behandlung, Zieldefinition und Durchführung der entsprechenden Methoden stellen zentrale Elemente dar und sind wesentliche Faktoren für das Gelingen einer Behandlung.

Die AVM freut sich, wenn viele Kolleginnen und Kollegen an der Tagung teilnehmen, und wünscht sich einen regen Austausch zu den Themen.

Für den Vorstand und das Tagungsteam

Univ.-Doz. Dr. Gerald Gatterer
Anmeldung und Informationen: Tagungsbüro der AVM: Paris-Lodron-Straße 32, 5020 Salzburg Tel.: +43/(0)662/88 41 66, Fax: +43/(0)662/88 6566 office@verhaltenstherapie-avm.at www.tagung-avm.at www.verhaltenstherapie-avm.at 


\section{Df기}

\section{Deutsche Ärztliche Gesellschaft für Verhaltenstherapie e.V. (DÄVT)}

\author{
Sekretariat DÄVT e.V. \\ Schön Klinik Roseneck \\ z.Hd. Frau Ruhstorfer \\ Am Roseneck 6, 83209 Prien am Chiemsee, Deutschland \\ info@daevt.de,www.daevt.de
}

\section{Einladung zum 3. IFA-Kongress: «Brücken bauen - Balint und IFA-Gruppen im Dialog»}

vom 15. bis 17. Juni 2018, Abteilung für Allgemeine

Psychosomatik, Klinik St. Irmingard in Prien am Chiemsee

Sehr geehrte Kolleginnen und Kollegen,

wir laden Sie ganz herzlich zu unserem inzwischen 3. IFA-Kongress, diesmal nach Prien am Chiemsee, ein. Der 1. IFA-Kongress fand 2014 in Berlin statt und wurde im Jahr 2016 vom 2. IFA-Kongress in Erfurt gefolgt. Wir haben interessante Begegnungen sowohl in den Klein- als auch in der Großgruppen erlebt und gemeinsam darüber gestaunt, was sich in den letzten 20 Jahren alles im Rahmen der interaktionsbezogenen Fallarbeit entwickelt hat.

Parallel dazu gibt es seit über 10 Jahren einen zunehmend intensiven Austausch mit den Kollegen der Deutschen Balint-Gesellschaft (DBG). Im Rahmen der Würzburger Balint-Tagung findet nunmehr seit gut 10 Jahren immer auch eine IFA-Gruppe statt. Auf dem DGPM-Kongress 2017 gab es ein erstes gemeinsames Symposium mit dem Titel: «Balint- und IFA-Gruppen im Dialog», das auf dem diesjährigen DGPM-Kongress 2018 zum zweiten Mal stattfinden wird. Diese Zusammenarbeit hat viel Vertrauen aufgebaut und ermöglicht, und so war es nur konsequent, dass wir auf Vorstandsebene der DBG, der Deutschen Ärztlichen Gesellschaft für Verhaltenstherapie (DÄVT) und des Verbandes für Integrative Verhaltenstherapie aus Lübben (VIVT) Kooperations- und gemeinsame Entwicklungsmöglichkeiten ausgelotet haben. Diese Gespräche und gemeinsamen Ziele sind inzwischen in einem Kooperationsvertrag zusammengefasst worden, der am 1. Januar 2018 in Kraft getreten ist. Ein Zeichen dieser Zusammenarbeit ist auch das erstmals gemeinsam gestaltete Balint-Journal 4/2017.

Im Rahmen dieses Kooperationsvertrags wird auf dem diesjährigen IFA-Kongress daher neben einem der beiden Eröffnungsvorträge auch erstmals eine Balint-Leitergruppe angeboten, in der die Möglichkeit für anerkannte IFA-Gruppenleiter besteht, insgesamt 10 Stunden an Balint-Leiterkompetenz zu erwerben. Diese Gruppe wird von Peter Stammberger (Stuttgart) geleitet, dem 2. Vorsitzenden der DBG.
Umgekehrt können von der DBG anerkannte Balint-Gruppenleiter im Rahmen der IFA-Leitergruppe ebenfalls 10 Fortbildungsstunden erwerben. Diese Gruppe wird von Herrn Dr. Rudolf Knickenberg (Aschaffenburg) gemeinsam mit Dr. Lars Thessen (Berlin) geleitet.

Neben den beiden Leitergruppen finden eine IFA-Anfängergruppe unter der Leitung von Christin Eichner und Holger Feiss, beide aus Berlin, sowie eine IFA-Fortgeschrittenengruppe unter der Leitung von Mechthild Kerkloh, Berlin, und Jens Nieswandt aus Stralsund statt.

\section{Programmablauf}

Freitag 15. Juni (große Turnhalle)

14:00 bis 14:15 Uhr $\quad$ Begrüßung durch den Geschäftsführer der Klinik St. Irmingard, Herrn Hämel, und Eröffnung durch die Veranstalter DÄVT (Dr. Ehrig) und VIVT (Herr Grünbaum)

Eröffnungsvorträge:

14:15 bis 15:00 Uhr

«Entwicklung und IFA-Gruppenarbeit» (Prof. Serge Sulz, München und Universität Eichstätt)

15:00 bis 15.45 Uhr «Die Dimensionen der Balintgruppenarbeit» (Dr. Peter Stammberger, Stuttgart, 2. Vorsitzender der DBG)

15:45 bis 16:15 Uhr Kaffeepause (kleine Turnhalle)

16:15 bis 17:45 Uhr Gruppenarbeit in 4 parallelen Gruppen:

- Balint-Leitergruppe

(P. Stammberger/Gesellschaftsraum)

- IFA-Leitergruppe (R. Knickenberg und

L. Theßen/Raum Fraueninsel)

- IFA-Teilnehmer (Anfänger) (C. Eichner und J. Nieswandt/Raum Herrenchiemsee)

- IFA-Teilnehmer (Fortgeschrittene)

(M. Kerkloh und M. Feiss/Raum Chiemsee)

17:45 bis 18:00 Uhr Kurzer Tagesabschluss/Organisatorisches 19:00 bis 21:00 Uhr Abendveranstaltung - gemütliches Zusammensein im See Cafe Toni (erreichbar mit PKW, Fahrrad oder zu Fuß; etwa 5 km)

Samstag 16. Juni

9:00 bis 10:45 Uhr

IFA-Gruppe im Forum (Großgruppe/ J. Grünbaum) (große Turnhalle)

10:45 bis $11: 15 \mathrm{Uhr}$ Kaffepause (kleine Turnhalle)

11:15 bis $12.00 \mathrm{Uhr}$ Vorträge (große Turnhalle): «Die Bedeutung des Ebenenwechsels in der IFA-Gruppenarbeit» (Dipl. Psych. Mechthild Kerkloh, Präsidentin der IFA-Gesellschaft VIVT, Berlin) 
12:00 bis 12:45 Uhr «Achtsamkeit und IFA-Gruppenarbeit» (Dr. med. Frank Udo Stepputat, Traunstein)

13:00 bis 14:00 Uhr Mittagessen (kleine Turnhalle)

14:30 bis 16:00 Uhr Gruppenarbeit in 4 parallelen Gruppen:

- Balint-Leitergruppe

(P. Stammberger/Gesellschaftsraum)

- IFA-Leitergruppe (R. Knickenberg und

L. Theßen/Raum Fraueninsel)

- IFA-Teilnehmer (Anfänger) (C. Eichner und J. Nieswandt/Raum Herrenchiemsee)

- IFA-Teilnehmer (Fortgeschrittene) (M. Kerkloh und M. Feiss/Raum Chiemsee)

16:00 bis 16:30 Uhr Kaffeepause (kleine Turnhalle)

16:30 bis 18:00 Uhr Gruppenarbeit in 4 parallelen Gruppen:

- Balint-Leiterguppe

(P. Stammberger/Gesellschaftsraum)

- IFA-Leitergruppe (R. Knickenberg und

L. Theßen/Raum Fraueninsel)

- IFA-Teilnehmer (Anfänger) (C. Eichner und J. Nieswandt/Raum Herrenchiemsee)

- IFA-Teilnehmer (Fortgeschrittene)

(M. Kerkloh und M. Feiss/Raum Chiemsee)

19:00 bis 22:00 Uhr Abendveranstaltung-gemeinsamer gemütlicher Ausklang im Wiener Kaffee der Klinik St. Irmingard

Sonntag 17. Juni (Große Turnhalle)

9:00 bis 10:45 Uhr

Balint-Gruppe im Forum

(Großgruppenarbeit/Stammberger)

10:45 bis 11:15 Uhr Kaffeepause (kleine Turnhalle)

11:15 bis 12:45 Uhr Gruppenarbeit in 4 parallelen Gruppen:

- Balint-Leitergruppe

(P. Stammberger/Gesellschaftsraum)

- IFA-Leitergruppe (R. Knickenberg und

L. Theßen/Raum Fraueninsel)

- IFA-Teilnehmer (Anfänger) (C. Eichner und J. Nieswandt/Raum Herrenchiemsee)

- IFA-Teilnehmer (Fortgeschrittene)

(M. Kerkloh und M. Feiss/Raum Chiemsee)

12:45 bis 13:15 Uhr Verabschiedung und Dank (große Turnhalle)

13:15 bis 14:00 Uhr Mittagessen (kleine Turnhalle)
In der Kongressgebühr sind die Teilnahme an allen 4 Vorträgen, der jeweils gewählten Gruppe, den beiden Demo-Gruppen im Forum sowie der Pausenversorgung und dem Mittagessen inklusive. An beiden Abenden gibt es die Möglichkeit zur Begegnung in entspannter Atmosphäre. Das See Cafe Toni befindet sich etwa $5 \mathrm{~km}$ von der Klinik entfernt und ermöglicht einen tollen Ausblick auf den See und die Bergsilhouette (www.seecafe-toni.de). Das Cafe ist entlang des Uferwegs zu Fuß oder mit dem Fahrrad ebenso gut zu erreichen wie mit dem Auto.

Am zweiten Abend besteht im Wiener Cafe in der Klinik die Möglichkeit zum gemütlichen Zusammensein bei diversen Getränken. Im Umfeld der Klinik gibt es ansonsten auch viele Restaurants mit sehr unterschiedlichen Angeboten.

Wir freuen uns auf anregende Gespräche und Begegnungen mit Ihnen sowie herrliches Sommerwetter. Vergessen Sie daher Ihre Badesachen nicht. Die Klinik liegt direkt am Chiemsee.

Mit kollegialen Grüßen

Dr. med. Christian Ehrig

Präsident DÄVT

Dipl. Psych. Johannes Grünbaum

1. Vorsitzender VIVT

Interessante Links:

www.tourismus.prien.de

www.st-irmingard.de

www.daevt.de

www.VIVT.de

www.Balintgesellschaft.de

www.cip-akademie.de

www.sbt-nord.de 